\title{
Typologie des investisseurs institutionnels et performance des firmes dans le contexte français
}

\author{
Jean Michel Sahut \\ Professeur et Directeur de la Recherche \\ HEG Genève et Cerege EA 1722 - Université de Poitiers \\ Hidaya Othmani Gharbi \\ Docteur en Sciences de Gestion \\ Faculté d'Economie Appliquée et CERGAM \\ Université Paul Cézanne - Aix Marseille III
}

\begin{abstract}
L'objet de cet article est d'analyser la relation entre les investisseurs institutionnels et la performance financière des firmes. Dans ce contexte, nous proposons une nouvelle typologie fondée sur la variété des comportements des institutionnels (passifs ou engagés (actifs)) vis-àvis de la gestion et du gouvernement des firmes qu'ils détiennent en portefeuille, et identifions les principaux facteurs pouvant influencer ces comportements. Nos résultats corroborent, en général, un impact positif de l'engagement des institutionnels sur la performance des firmes. Plus précisément, ils montrent que l'influence des investisseurs institutionnels sur la performance financière des firmes dépend des facteurs d'influence de leur comportement, et de l'existence d'une relation bidirectionnelle entre ces différents types d'investisseurs institutionnels et la performance.
\end{abstract}

Mots clés : investisseurs institutionnels - performance - typologie - passivité - engagement.

\section{Institutional investors' typology and firm performance in the French context}

The main purpose of this paper is to analyse the relation between institutional ownership and firm financial performance. We propose in this context a new typology of institutional investors based on their behaviours (active or passive) and the principal factors that may influence them. Globally, our results corroborate a positive impact of institutional activism on firm performance. Specially, they support that the effects of institutional owners on firm performance depend on their behaviours and that institutional active behaviour is more apparent with the grouping of its influential factors. We also find that the relation between institutional ownership and firm performance is bilateral.

Key worlds: institutional investors - firm performance - typology - passive behaviour -active behaviour. 
La séparation des fonctions de propriété et de contrôle au sein des entreprises ainsi que ses implications sur les décisions stratégiques et la performance sont au centre des débats de la recherche sur la gouvernance depuis des décennies. Ces débats ont été significativement marqués par les apports des théories de l'agence et du gouvernement des entreprises (Jensen et Meckling, 1976 ; Demsetz, 1983 ; Schleifer, Vishny, 1986, etc.) qui ont étudié les structures de propriété et de contrôle afin d'analyser et de montrer comment réduire les conflits d’intérêts entre les différentes parties prenantes de la firme. En particulier, l'étude des aspects de la structure de propriété comme la concentration de la propriété, et l’identité des actionnaires ont permis d'apporter un regard nouveau sur les systèmes de gouvernement des firmes, et la création de la valeur actionnariale.

Dans ce travail de recherche, nous nous intéressons à l'impact d'un type particulier d’investisseurs, les investisseurs institutionnels, sur la performance.

Cette relation a généré une importante littérature aux conclusions souvent contradictoires. D’autres investigations s’avèrent donc nécessaires pour clarifier la relation propriété performance financière, surtout dans le contexte mondial actuel de crise.

Cette recherche y répond en partie car elle porte sur la période allant de juillet 2006 à juillet 2008. Cette période d'étude ne comprend donc pas le deuxième choc de la crise des « subprimes » intervenu le 15 septembre 2008 avec la faillite de la banque d’affaires Lehman Brothers, et l'intervention des états dans le capital et la gouvernance des banques. Nous estimons dans ce cas que les résultats de cette recherche sont limités à un environnement économique et financier relativement stable puisque la chute des actifs financiers s’est réellement accélérée après la faillite de cette Banque et la récession qui a été officiellement annoncée en France en octobre 2008. Ainsi, par rapport à la littérature existante, nous cherchons à :

- Déterminer les effets des investisseurs institutionnels sur la performance des firmes en fonction de leurs comportements (engagés (actifs) ou passifs), et les facteurs qui les influencent, dans le contexte français. Nous mettons ainsi l'accent sur l’hétérogénéité de ces 
acteurs contrairement à la majorité des travaux antérieurs qui les considèrent comme un groupe homogène,

- identifier la direction de la relation entre l'actionnariat institutionnels et la performance. A notre connaissance, très peu de travaux ont examiné cette relation bidirectionnelle (Tsai, Gu, 2007). La prise en compte de cet aspect constitue le principal apport de notre recherche.

Pour traiter ces différents aspects, notre recherche s’organise comme suit. Nous commençons par présenter notre nouvelle typologie des investisseurs institutionnels et les principaux facteurs qui les influencent. Nous exposons ensuite une revue de la littérature sur la relation entre l'actionnariat institutionnels et la performance, tout en élaborant nos hypothèses à tester. Notre troisième section concerne l'identification des modèles et des variables. Nous récapitulons l'ensemble des résultats trouvés dans la quatrième section, pour conclure à la fin.

\section{Variété des comportements et hétérogénéité des investisseurs institutionnels}

Suite à la croissance des participations des investisseurs institutionnels dans le capital des firmes, une importante littérature s’est développée afin de mieux comprendre leurs comportements et leurs influences sur la gouvernance des entreprises. Toutefois, la majorité de ces travaux traitent les investisseurs institutionnels comme un groupe homogène affichant les mêmes objectifs et agissant de la même manière. En pratique, ces acteurs sont loin de former un tel groupe homogène compte tenu de leurs environnements, objectifs et contraintes lesquels orientent leurs actions et plus généralement les modes de gestion de leurs investissements. Une analyse spécifique selon la typologie de ces investisseurs s’avère donc d’un apport non négligeable pour une discussion plus approfondie du lien entre leurs actions et la performance des firmes.

\subsection{Comportements des investisseurs institutionnels : de la passivité à l'engagement}


Nous distinguons principalement deux types d'investisseurs institutionnels, passifs ou engagés (actifs), en fonction de leur degré d’implication dans le gouvernement et la gestion en général des entreprises.

Un premier courant de recherche suppose généralement l'absence de toute incitation de ces acteurs à s’impliquer dans la gestion des entreprises où ils placent leurs fonds (Black, 1990 ; Bushee, 1998 ; et Dong, Ozkan, 2008). Ces derniers adoptent un comportement « passif », et s’intéressent essentiellement aux rendements à court terme de leurs titres.

A l'inverse, un comportement engagé ou actif de ces investisseurs a été appuyé par plusieurs travaux. Notons que le terme 'activisme’ est souvent utilisé pour qualifier le comportement des investisseurs institutionnels qui interfère dans la gestion et la gouvernance des firmes. Ce rôle suggère l'utilisation de leurs droits de vote pour participer au processus de prise de décision, et influencer les orientations des firmes dans le but de la maximisation de valeur.

Entre ces deux comportements passifs ou actifs, les débats sont nombreux et les résultats empiriques souvent contrastés ne permettent pas de trancher quant au rôle joué par ces investisseurs. Afin de mieux comprendre ces comportements, nous proposons de s'intéresser aux principaux facteurs, inspirés de la littérature, qui conditionnent de telles orientations. Ils constituent les caractéristiques essentielles de la nouvelle typologie que nous proposons.

\subsection{Facteurs d'influence des comportements des institutionnels}

Les deux principaux comportements déjà évoqués constituent, à notre sens, une source de différenciation principale entre les investisseurs institutionnels. En réalité, l'adoption de l’un de ces comportements est conditionnée par différents facteurs. A partir des travaux de Brickley et al. (1988), Kochhar, David (1996), Maug (1998) et Chen et al. (2007), nous avons identifié trois principaux facteurs que nous estimons être les plus influents. Nous considérons, comme première source de différenciation des investisseurs institutionnels, leurs horizons d'investissement qui peuvent varier du court au long terme, et influencer en conséquence leur 
comportement soit vers la passivité ou «myopie» (Bushee, 1998 ; Dong, Ozkan, 2008), ou plutôt vers l’engagement (Kochhar, David, 1996 ; Chen et al. 2007).

Le second facteur est le niveau de participation des institutionnels dans les structures de capital des firmes variant de la détention d'un nombre faible de titres, à la gestion de positions majoritaires. Nous suggérons alors que leur niveau de participation peut varier leurs orientations et en conséquence leurs comportements vers l'engagement ou la passivité (Khan et al. 2005).

Le troisième et dernier facteur concerne la nature des relations liant les institutionnels aux firmes en portefeuille. Nous distinguons dans ce cadre soit l'unique relation d'investissement, comme c'est le cas par exemple pour les fonds de pensions ou les fonds mutuels; ou le développement possible de relations d'affaires à côté de la relation d'investissement, comme les compagnies d'assurances ou encore les banques. Pour ces derniers, l'existence d'une éventuelle relation d'affaires peut les amener à la privilégier, au détriment de sa relation d'investissement, ce qui conduit à affaiblir le contrôle exercé sur la firme contrairement aux institutions ayant uniquement des relations d'investissement (Brickley et al. 1988 ; Kochhar, David, 1996 ; Cornett et al. 2007).

De plus, il existe certains liens entre ces trois facteurs, ou du moins au niveau de leur impact sur la performance qui semblent aller dans le même sens. Cette influence sur la performance fait l'objet de la section suivante.

\section{Actionnariat institutionnels et performance des firmes}

La variété des comportements des investisseurs institutionnels, concernant le gouvernement et la gestion en général des firmes, a amené de nombreux chercheurs à s’interroger sur l’impact direct de l'actionnariat institutionnels sur la performance financière des entreprises. Il s'agit dans ce cadre d'évaluer l'efficacité des comportements de ces acteurs sur la création de la richesse pour tous les actionnaires. 


\subsection{Relation entre les investisseurs institutionnels et la performance : cadres théoriques et validations empiriques}

Dans la littérature financière, l’influence de la présence des investisseurs institutionnels sur la performance des firmes peut se traduire positivement, négativement ou n’avoir aucun effet. Les principales hypothèses théoriques développées et leurs mises en évidence empiriques regroupent essentiellement :

- l'hypothèse du contrôle efficace, laquelle associe la présence des investisseurs institutionnels à une amélioration de la performance des firmes. Ces acteurs interviennent, dans ce cas, pour réduire les conflits d’intérêts entre les équipes dirigeantes et les autres actionnaires. Plusieurs études empiriques ont corroboré ce lien positif, à l'instar des travaux de McConnell, Servaes (1990), et de Cornett et al. (2008),

- les hypothèses de conflits d'intérêts et d'alignement stratégique soutenant un affaiblissement de la performance des firmes suite à la présence des institutionnels (Pound, 1988). En effet, l'existence de relations d'affaires profitables, à côté des relations d’investissement, pousse les actionnaires institutionnels à se retrouver en situation conflictuelle qui est généralement associée à un abaissement d’un contrôle rigoureux exercé par ces derniers.

Parallèlement, selon l'hypothèse de l'alignement stratégique, les investisseurs institutionnels peuvent constater qu’il est plus avantageux pour eux de coopérer avec les équipes dirigeantes des firmes, au détriment des autres actionnaires. La réduction de la valeur est donc une conséquence envisageable de cet alignement stratégique. Cette relation négative a d’ailleurs été identifiée empiriquement dans les travaux d’Alexandre, Paquerot (2000) pour le cas français, et d’Atanasov (2005) dans le contexte bulgare,

- l’hypothèse de la neutralité, laquelle stipule l'absence d'une influence de l'actionnariat institutionnels (et de la structure de propriété en général) sur la performance des firmes (Demsetz, 1983). Empiriquement, cet effet neutre de l'actionnariat institutionnel sur la 
performance a été renforcé par plusieurs recherches à l'instar de celles d'Agrawal, Knoeber (1996) et Mînguez-Vera, Martin-Ugedo (2007) entre autres.

En plus de ces trois hypothèses issues directement de la littérature, nous proposons d'étudier un quatrième point concernant la possibilité d'une relation non linéaire entre l'actionnariat institutionnels et la performance. Notre réflexion a pour origine les travaux de Morck et al. (1988), et Mcconnell, Servaes (1990) qui démontrent l'existence d'une relation non linéaire entre la performance et la structure de propriété, à travers ses deux dimensions la concentration de la propriété et l’actionnariat de l’équipe dirigeante.

Ainsi, ces divers débats montrent qu'il est difficile de conclure quant à la nature de liens possibles entre l'actionnariat institutionnels et la performance. De plus, ces travaux ont considéré les investisseurs institutionnels comme un groupe homogène, ce qui, à notre sens, pourrait expliquer en partie les résultats mixtes trouvés. Par conséquent, nous mettons l'accent dans nos hypothèses sur le caractère hétérogène des institutionnels. L’ajout de ce caractère aux hypothèses plus classiques nous amène à suggérer que l'effet de ces investisseurs sur la performance varie en fonction de leur comportement actif ou passif. Ainsi, nous supposons :

H1 : Un effet négatif des investisseurs institutionnels orientés vers l'adoption d'un comportement passif sur la performance.

A partir de cette hypothèse principale, nous distinguons :

H1a : La dispersion des actionnaires institutionnels favorise un comportement passif. Ils auront une possible influence négative sur la performance.

H1b: Les actionnaires institutionnels développant une dualité de relations (affaire et investissement) avec les firmes en portefeuille adoptent davantage un comportement passif. Ils ont un impact négatif ou non linéaire sur la performance.

H2 : Les actionnaires institutionnels orientés vers l'adoption d'un comportement engagé (actif) favorisent l'amélioration de la performance des firmes. 
Nous proposons dans ce cas :

H2a : Une concentration de l'actionnariat institutionnel encourage une orientation active de ces acteurs qui est associée à une amélioration de la performance.

H2b : Une présence des institutionnels maintenant uniquement des relations d'investissement avec les firmes en portefeuille favorise un comportement actif de ces derniers. Leur impact sur la performance est positif ou non linéaire.

H2c: Une relation positive entre l'actionnariat institutionnel et la performance est plus significative lorsque les principaux facteurs d’influence d'un comportement actif sont réunis.

\subsection{Endogénéité de la variable actionnariat institutionnels}

La question de l'endogénéité de la structure de propriété est l'une des principales problématiques de la littérature sur la gouvernance. En effet, en étudiant la nature de la relation entre la structure de propriété et la performance, nombreuses recherches ont implicitement assumé le caractère uniquement exogène des variables de la structure de propriété. En ce sens, une seule direction de la relation a été examinée. Par opposition, d'autres études ont appuyé un caractère plutôt endogène des variables de la structure de propriété (Demsetz, Villalonga (2001); Switzer, Kelly, 2006). Ainsi, la relation entre la structure de propriété et la performance semble plutôt être bilatérale. Par conséquent, une vérification de cette relation dans les deux sens possibles s’impose.

Ainsi, nous émettons l'hypothèse suivante :

H3 : Une relation bidirectionnelle existe entre les investisseurs institutionnels et la performance des firmes.

\section{Modèles et variables}

Notre échantillon repose sur les entreprises de l’indice SBF120 pour la période 2006-2008, soit un panel totalisant 337 observations (société/année). Les données relatives à l’actionnariat 
institutionnel ont été obtenues à partir de la base de données Reuters, et les données financières des bases Worldscope et Thomson Financial.

\subsection{Spécifications des modèles}

Afin de tester l'influence de la typologie proposée des institutionnels sur la performance, et en tenant compte d'une possible relation bidirectionnelle entre ces variables, nous spécifions le système d'équations suivantes. Précisons qu’au vu des débats théoriques et empiriques, nous vérifions aussi bien l'approche linéaire que non linéaire (de forme quadratique) afin d’identifier le modèle le plus significatif.

- Modèle 1 (M1) : $\quad$ PERF= fonction (INST, Variables explicatives)

- Modèle 2 (M2) : PERF= fonction (INST, INST ${ }^{2}$, Variables explicatives)

- Modèle 3 (M3) : $\quad$ INST= fonction (PERF, Variables explicatives)

- Modèle 4 (M4) : INST= fonction (PERF, PERF ${ }^{2}$, Variables explicatives)

INST : variables relatives à la typologie des institutionnels

INST $^{2}$ : carré des variables relatives à la typologie des institutionnels

PERF : Mesures de la performance de l'entreprise

$P E R F^{2}$ : Carré de la mesure de performance

En se référant principalement aux recherches de Demsetz, Villalonga (2001), Switzer, Kelly (2006) et Tsai, Gu (2007), nous appliquons aux différents modèles le test d'erreur de spécification de Hausman (1978) afin de spécifier la méthode d’estimation la plus appropriée (MCO, DMC) et éviter en conséquence tout risque de biais de nos résultats.

\subsection{Variables à tester}

Mesure de performance : La plupart des études utilisent des mesures hybrides dont le Q de Tobin qui est le plus adopté du fait de sa capacité à refléter les opportunités de croissance de l'entreprise. Nous utilisons dans cette recherche une mesure approximée du Q de Tobin (Q) qui est calculée par le rapport entre la somme de la valeur de marché des actions, la valeur comptable des dettes à long, moyen et court terme, et le total des actifs (Mîngez-Vera, MartinUgedo, 2007). 
Mesures de l'actionnariat institutionnels: En se basant sur notre typologie, nous proposons

les mesures suivantes pour ces acteurs :

Tab. 1 : Mesures de la typologie des institutionnels

\begin{tabular}{|c|l|c|}
\hline Typologie des institutionnels & \multicolumn{1}{|c|}{ Mesures utilisées } & Abréviations \\
\hline $\begin{array}{c}\text { Institutionnels ayant une dualité } \\
\text { de relations (désignés par } \\
\text { sensibles aux pressions) }\end{array}$ & $\begin{array}{l}\text { Pourcentage du capital d'une firme détenu par les } \\
\text { institutionnels avec une éventuelle dualité de relations } \\
\text { (les compagnies d'assurances, les banques possédant } \\
\text { aumoins 1\% du capital d'une firme) }\end{array}$ & SENS \\
\hline $\begin{array}{c}\text { Concentration de l'actionnariat } \\
\text { des institutionnels }\end{array}$ & $\begin{array}{l}\text { Pourcentage du capital détenu par les institutionnels } \\
\text { détenant des blocs de contrôle (au moins 5\% du } \\
\text { capital) dans une même firme. }\end{array}$ & BLOC \\
\hline $\begin{array}{c}\text { Institutionnels avec l'unique } \\
\text { relation d'investissement } \\
\text { (désignés par résistants aux } \\
\text { pressions) }\end{array}$ & $\begin{array}{l}\text { Pourcentage du capital d'une firme détenu par les } \\
\text { institutionnels avec uniquement une relation } \\
\text { d'investissement (les fonds de pension et } \\
\text { d'investissement et les fondations possédant au moins } \\
1 \% \text { du capital d'une firme) }\end{array}$ & \\
\hline \hline $\begin{array}{c}\text { Institutionnels réunissant le } \\
\text { maximum de facteurs d'influence } \\
\text { d'un comportement actif }\end{array}$ & $\begin{array}{l}\text { Blocs de contrôle des institutionnels résistants aux } \\
\text { pressions ayant des horizons de long terme }\end{array}$ & BLOCLTINSENS \\
\hline
\end{tabular}

Pour les institutionnels regroupant le maximum de facteurs d'influence d'un comportement engagé, nous considérons ceux résistants aux pressions, détenant des blocs de contrôle, et ayant un horizon d'investissement à long terme (BLOCLTINSENS). Pour la définition de ces horizons, nous procédons de la même manière que Chen et al. (2007), en vérifiant si ces principaux actionnaires institutionnels ont maintenu ou augmenté leur participation durant une année (soit pour 2007 et 2008 du fait des données disponibles).

Variables explicatives: Le tableau suivant récapitule l'ensemble des variables explicatives

Tab.2: Variables explicatives utilisées

\begin{tabular}{|c|c|c|}
\hline Variables explicatives & Nom complet & Définition \\
\hline \multicolumn{2}{|c|}{ Pour les équations à variable expliquée la Performance } \\
\hline 1-LOGTA & Taille de l'entreprise & $\begin{array}{c}\text { le logarithme du total des actifs d'une } \\
\text { entreprise }\end{array}$ \\
\hline 2-DETTE & Endettement & \begin{tabular}{c} 
Dettes à long terme \\
\cline { 2 - 3 }
\end{tabular} \\
\hline 3-RD & $\begin{array}{c}\text { Dépenses en recherche et } \\
\text { développement }\end{array}$ & \begin{tabular}{c} 
Dépenses en Recherches et Développement \\
\cline { 2 - 3 }
\end{tabular} \\
\hline \multicolumn{3}{|c}{ Pour les équations à variable expliquée l'actionnariat institutionnels } \\
\hline
\end{tabular}




\begin{tabular}{|c|c|c|}
\hline 2-LIQUID & Liquidité & entreprise \\
\hline 3-BETA & Risque systématique & $\begin{array}{c}\text { volume annuel des actions échangées } \\
\text { le nombre total des titres d'une entreprise } \\
\text { estimé à partir du MEDAF en utilisant des } \\
\text { rendements journaliers pour chaque année } \\
\text { d'étude }\end{array}$ \\
\hline 4-RSPECIFIQUE & Risque spécifique & $\begin{array}{c}\text { Ecart type relatif aux résidus du MEDAF } \\
\text { annualisé en le multipliant par la racine carrée } \\
\text { de 252 (nombre de jours de cotation par an) }\end{array}$ \\
\hline 5-DIVID & Dividende & dividende par action \\
\hline
\end{tabular}

\section{Résultats empiriques}

Notre analyse de la relation entre actionnariat institutionnels et performance des firmes nous a amené à formuler plusieurs hypothèses que nous testons empiriquement dans cette partie.

\subsection{Concentration de l'actionnariat institutionnels et performance des firmes}

Nous avons considéré la concentration de l'actionnariat institutionnels comme étant un premier facteur pouvant favoriser un comportement engagé ou actif de ces derniers. Nos résultats sont en ligne avec ces propositions pour des niveaux de concentration des institutionnels au delà de 32\% (point d'inflexion pour la relation non linéaire, modèle M2), confirmant ainsi en partie notre hypothèse H2a. Pour des participations réduites des institutionnels, soit le cas de la dispersion de leur actionnariat, l'effet est négatif sur la performance, ce qui corrobore notre hypothèse H1a. Nous estimons donc qu'avec l'accroissement de la participation des institutionnels, il devient plus avantageux pour eux d'exercer un contrôle plus rigoureux, et favoriser en conséquence l'amélioration de la performance.

Notre hypothèse H3, spécifiant une relation bidirectionnelle entre la concentration de l’actionnariat des institutionnels et la performance, est également validée.

Tab.3 : Performance et concentration de la propriété des institutionnels

\begin{tabular}{|l|l|l|l|l|}
\hline \multirow{2}{*}{} & \multicolumn{2}{|c|}{ Q } & \multicolumn{2}{|c|}{ BLOC } \\
\cline { 2 - 5 } & M1 & M2 & M3 & M4 \\
\hline
\end{tabular}




\begin{tabular}{|c|c|c|c|c|}
\hline Constante & $\begin{array}{c}4.916^{* * *} \\
(13.4)\end{array}$ & $\begin{array}{c}5.005^{* * *} \\
(13.69)\end{array}$ & $\begin{array}{l}14.198 \\
(1.36)\end{array}$ & $\begin{array}{c}30.115^{* * *} \\
(2.625)\end{array}$ \\
\hline BLOC & $\begin{array}{c}-0.0038 \\
(-1.47)\end{array}$ & $\begin{array}{c}-0.0213^{* * *} \\
(-2.928)\end{array}$ & & \\
\hline $\mathrm{BLOC}^{2}$ & & $\begin{array}{c}0.00033^{* * *} \\
(2.57)\end{array}$ & & \\
\hline $\mathrm{Q}$ & & & $\begin{array}{l}-1.97 * * \\
(-1.933)\end{array}$ & $\begin{array}{c}-8.689 * * * \\
(-3.628)\end{array}$ \\
\hline$\overline{Q^{2}}$ & & & & $\begin{array}{c}0.996 * * * \\
(3.091)\end{array}$ \\
\hline TAILLE & $\begin{array}{c}-0.233 * * * \\
(-10.148)\end{array}$ & $\begin{array}{c}-0.235 * * * \\
(-10.31)\end{array}$ & $\begin{array}{c}-0.725 \\
(-1.118)\end{array}$ & $\begin{array}{c}-1.262 * * \\
(-1.903)\end{array}$ \\
\hline DETTE & $\begin{array}{l}-0.0007 \\
(-0.245)\end{array}$ & $\begin{array}{c}-0.00018 \\
(-0.06)\end{array}$ & & \\
\hline $\mathrm{RD}$ & $\begin{array}{c}0.041^{* * *} \\
(3.246)\end{array}$ & $\begin{array}{l}0.04^{* * *} \\
(3.206)\end{array}$ & & \\
\hline LIQUIDITE & & & $\begin{array}{c}-0.176 \\
(-0.568)\end{array}$ & $\begin{array}{c}-0.263 \\
(-0.853)\end{array}$ \\
\hline BETA & & & $\begin{array}{c}4.5 \\
(1.4)\end{array}$ & $\begin{array}{c}3.985 \\
(1.257)\end{array}$ \\
\hline RSPECIFIQUE & & & $\begin{array}{c}0.183^{* * *} \\
(2.387)\end{array}$ & $\begin{array}{l}0.166^{* *} \\
(2.198)\end{array}$ \\
\hline DIVID & & & $\begin{array}{c}1.933^{* * *} \\
(2.86)\end{array}$ & $\begin{array}{c}1.852 * * * \\
(2.773)\end{array}$ \\
\hline$\overline{\mathrm{R}^{2}}$ & 0.276 & 0.29 & 0.055 & 0.082 \\
\hline $\begin{array}{c}\text { F - statistic } \\
\text { (P-value) }\end{array}$ & $\begin{array}{c}25.012 \\
(0.00)\end{array}$ & $\begin{array}{l}22.298 \\
(0.00)\end{array}$ & $\begin{array}{l}3.156 \\
(0.00)\end{array}$ & $\begin{array}{l}4.141 \\
(0.00)\end{array}$ \\
\hline
\end{tabular}

* Significatif au seuil de $10 \% * *$ significatif au seuil de $5 \% * * *$ significatif au seuil de $1 \%$

\subsection{Nature des relations développées et performance des firmes}

Pour les institutionnels développant une unique relation d'investissement (INSENS), les résultats corroborent notre hypothèse H2b. En effet, une relation non linéaire est identifiée entre ces acteurs et le Q de Tobin indiquant :

-un effet négatif de la dispersion des INSENS sur la performance (comportement passif).

-un effet positif pour une détention des INSENS proche de la minorité de blocage (à partir d'un seuil de 32.8\%, modèle M2), ce qui confirme, dans ce cas, l'orientation active et le contrôle efficace de ces acteurs. Ces résultats sont également en adéquation avec ceux de Kochhar, David, 1996 ; et Cornett et al., 2007. De plus, cette relation non linéaire est bidirectionnelle entre les institutionnels résistants aux pressions et le Q de Tobin.

Pour les institutionnels sensibles aux pressions (SENS), une relation non linéaire est également identifiée avec le Q de Tobin, mais avec un point d’inflexion légèrement supérieur à celui des institutionnels résistants aux pressions (INSENS), soit 35.5\%. 
Nous remarquons que pour des participations réduites des investisseurs institutionnels, c’est plutôt l'orientation passive qui domine, et ce quelque soit la nature des relations qu'ils peuvent développer avec les firmes. Par contre, l'accroissement de l'actionnariat des institutionnels, sensibles ou résistants aux pressions, favorise une amélioration de la performance.

Nous suggérons donc que cette distinction des institutionnels selon les types de relations, dans le contexte français, n’est pas d'une grande utilité contrairement aux études américaines. Nous estimons que plusieurs raisons sont à l'origine d'un tel constat :

-l'existence d'investisseurs institutionnels de divers continents, en l'occurrence français et étrangers qui n’ont ni les mêmes objectifs, contraintes ou modes de rémunération, en particulier pour les hedges funds (leur rémunération dépend de la performance et pas du montant des actifs gérés),

-les faibles fonds investis dans les firmes françaises par les institutionnels anglo-saxons, et surtout les fonds de pension et d'investissements américains, par rapport au volume des portefeuilles qu’ils gèrent. Le montant limité de ces investissements réduit en conséquence leurs incitations à intervenir activement dans le contrôle de ces firmes.

Tab.4 : Performance et présence des institutionnels résistants aux pressions.

\begin{tabular}{|c|c|c|c|c|}
\hline & \multicolumn{2}{|c|}{$\mathrm{Q}$} & \multicolumn{2}{|c|}{ INSENS } \\
\hline & M1 & M2 & M3 & M4 \\
\hline Constante & $\begin{array}{c}5.074 * * * \\
(13.21)\end{array}$ & $\begin{array}{c}5.24^{* * *} \\
(13.73)\end{array}$ & $\begin{array}{c}43.58 * * * \\
(4.64)\end{array}$ & $\begin{array}{c}56.65 * * * \\
(5.45)\end{array}$ \\
\hline INSENS & $\begin{array}{c}-0.0047 * \\
(-1.67) \\
\end{array}$ & $\begin{array}{c}-0.0296 * * * \\
(-3.687)\end{array}$ & & \\
\hline INSENS $^{2}$ & & $\begin{array}{c}0.00045^{* * *} \\
(3.305)\end{array}$ & & \\
\hline $\mathrm{Q}$ & & & $\begin{array}{l}-1.501^{*} \\
(-1.625)\end{array}$ & $\begin{array}{c}-7.015^{* * * *} \\
(-3.233)\end{array}$ \\
\hline $\mathrm{Q}^{2}$ & & & & $\begin{array}{c}0.818^{* * *} \\
(2.802)\end{array}$ \\
\hline TAILLE & $\begin{array}{c}-0.242 * * * \\
(-10.33)\end{array}$ & $\begin{array}{c}-0.243^{* * *} \\
(-10.49)\end{array}$ & $\begin{array}{c}-1.945 * * * \\
(-3.321)\end{array}$ & $\begin{array}{c}-2.386 * * * \\
(-3.973)\end{array}$ \\
\hline DETTE & $\begin{array}{l}-0.0011 \\
(-0.377) \\
\end{array}$ & $\begin{array}{c}-0.001 \\
(-0.331) \\
\end{array}$ & & \\
\hline $\mathrm{RD}$ & $\begin{array}{c}0.041^{* * *} \\
(3.21)\end{array}$ & $\begin{array}{c}0.0407 * * * \\
(3.264) \\
\end{array}$ & & \\
\hline LIQUIDITE & & & $\begin{array}{c}1.471^{* * *} \\
(5.228)\end{array}$ & $\begin{array}{c}1.399 * * * \\
(5.008)\end{array}$ \\
\hline
\end{tabular}




\begin{tabular}{|c|c|c|c|c|}
\hline BETA & & & -3.168 & -3.59 \\
& & & $-0.187)$ & $(-1.25)$ \\
\hline RSPECIFIQUE & & & 0.039 \\
$(0.571)$ & 0.026 \\
& & & -0.114 & $(0.381)$ \\
\hline DIVID & & & $-0.187)$ & -0.182 \\
& & & 0.127 & 0.148 \\
\hline $\mathrm{R}^{2}$ & 0.277 & 0.301 & 7.891 & 8.029 \\
\hline F- statistic & 25.18 & 23.438 & $(0.00)$ & $(0.00)$ \\
\hline
\end{tabular}

* Significatif au seuil de $10 \%$ ** significatif au seuil de $5 \%$ *** significatif au seuil de $1 \%$

Tab.5 : Performance et présence des institutionnels sensibles aux pressions.

\begin{tabular}{|c|c|c|c|c|}
\hline & \multicolumn{2}{|c|}{$\mathrm{Q}$} & \multicolumn{2}{|c|}{ SENS } \\
\hline & M1 & M2 & M3 & M4 \\
\hline Constante & $\begin{array}{c}4.826 * * * \\
(13.12)\end{array}$ & $\begin{array}{c}4.829 * * * \\
(13.24)\end{array}$ & $\begin{array}{c}10.561 \\
(0.7)\end{array}$ & $\begin{array}{l}23.406 \\
(1.318)\end{array}$ \\
\hline SENS & $\begin{array}{l}-0.005 \\
(-1.48)\end{array}$ & $\begin{array}{c}-0.027 * * * \\
(-2.889)\end{array}$ & & \\
\hline SENS $^{2}$ & & $\begin{array}{c}0.00038^{* *} \\
(2.516) \\
\end{array}$ & & \\
\hline $\mathrm{Q}$ & & & $\begin{array}{l}-4.06^{*} \\
(-1.6)\end{array}$ & $\begin{array}{c}-12.297 * * \\
(-2.039)\end{array}$ \\
\hline $\mathrm{Q}^{2}$ & & & & $\begin{array}{c}1.637^{*} \\
(1.6)\end{array}$ \\
\hline TAILLE & $\begin{array}{c}-0.227 * * * \\
(-9.689) \\
\end{array}$ & $\begin{array}{c}-0.221 * * * \\
(-9.46) \\
\end{array}$ & $\begin{array}{c}-0.474 \\
(-0.599) \\
\end{array}$ & $\begin{array}{c}-0.756 \\
(-0.899) \\
\end{array}$ \\
\hline DETTE & $\begin{array}{l}-0.0006 \\
(-0.213)\end{array}$ & $\begin{array}{c}-0.00018 \\
(-0.06)\end{array}$ & & \\
\hline $\mathrm{RD}$ & $\begin{array}{l}0.04^{* * *} \\
(3.183) \\
\end{array}$ & $\begin{array}{c}0.039 * * * \\
(3.063)\end{array}$ & & \\
\hline LIQUIDITE & & & $\begin{array}{c}-0.441 * * \\
(-1.83)\end{array}$ & $\begin{array}{c}-0.579 * * \\
(-2.184)\end{array}$ \\
\hline BETA & & & $\begin{array}{l}7.71^{* *} \\
(3.053)\end{array}$ & $\begin{array}{c}6.296 * * \\
(2.268)\end{array}$ \\
\hline RSPECIFIQUE & & & $\begin{array}{l}0.082 \\
(1.35)\end{array}$ & $\begin{array}{c}0.066 \\
(1.043) \\
\end{array}$ \\
\hline DIVID & & & $\begin{array}{c}1.965^{* * * *} \\
(3.594)\end{array}$ & $\begin{array}{c}2.102^{* * *} \\
(3.667)\end{array}$ \\
\hline $\mathrm{R}^{2}$ & 0.276 & 0.29 & 0.095 & 0.032 \\
\hline $\begin{array}{c}\mathrm{F} \text { - statistic } \\
\text { (P-value) }\end{array}$ & $\begin{array}{l}25.017 \\
(0.00)\end{array}$ & $\begin{array}{l}22.24 \\
(0.00) \\
\end{array}$ & $\begin{array}{l}6.745 \\
(0.00) \\
\end{array}$ & $\begin{array}{l}6.172 \\
(0.00) \\
\end{array}$ \\
\hline
\end{tabular}

* Significatif au seuil de $10 \% * *$ significatif au seuil de $5 \% * * *$ significatif au seuil de $1 \%$

\subsection{Réunion des facteurs d'influence d'un comportement engagé et performance}

Nos hypothèses théoriques suggèrent qu'un comportement actif des investisseurs institutionnels est plus probable avec l'augmentation du nombre de facteurs qui peuvent l'influencer. L'identification de ce comportement actif peut par conséquence influencer positivement la performance des firmes (hypothèse H2c). Nos résultats présentés dans le tableau 6 confirment ces constats. Ainsi, la détention de blocs d'actions par les institutionnels résistants aux pressions et ayant un horizon d'investissement de long terme 
(BLOCLTINSENS) influence positivement le Q de Tobin et ce à partir d'un niveau d'actionnariat de 17\% (modèle M2). Ce niveau est beaucoup plus faible que les niveaux constatés en étudiant les facteurs d’influence séparément (réduction de près de la moitié).

En proposant d'encore affiner ces résultats, nous avons retenu à partir de notre échantillon initial uniquement les firmes vérifiant au moins l'existence d'un BLOCLTINSENS ${ }^{1}$ (nous éliminons ainsi les entreprises à position nulle). En examinant ce sous échantillon, les régressions obtenues précisent nos observations. Nous retenons principalement la relation positive et significative entre BLOCLTINSENS et Q, indiquant que ce type d'institutionnels participe effectivement à la création de la valeur. Ce résultat affirme le comportement actif de ces acteurs et leur participation et implication efficace dans le gouvernement et la gestion des firmes.

De plus, nous constatons un effet positif et linéaire (significatif à 10\%) du Q de Tobin sur la variable BLOCLTINSENS, appuyant ainsi la thèse de la recherche de gains à long terme et du développement de relations durables pour ce type d'investisseurs.

Tab.6 : Performance et institutionnels regroupant les trois facteurs d'influence d'un comportement actif.

\begin{tabular}{|c|c|c|c|c|}
\hline & \multicolumn{2}{|c|}{$\mathrm{Q}$} & \multicolumn{2}{|c|}{ BLOCLTINSENS } \\
\hline & M1 & M2 & M3 & M4 \\
\hline Constante & $\begin{array}{c}4.854 * * * \\
(10.264)\end{array}$ & $\begin{array}{l}5.024 * * * \\
(10.954)\end{array}$ & $\begin{array}{c}2.94 \\
(0.209) \\
\end{array}$ & $\begin{array}{l}10.588 \\
(0.611) \\
\end{array}$ \\
\hline BLOCLTINSENS & $\begin{array}{l}0.0026 \\
(0.494)\end{array}$ & $\begin{array}{c}-0.0505^{* * * *} \\
(-3.639)\end{array}$ & & \\
\hline BLOCLTINSENS $^{2}$ & & $\begin{array}{c}0.00148^{* * *} \\
(4.139)\end{array}$ & & \\
\hline Q & & & $\begin{array}{l}3.999 * \\
(1.71)\end{array}$ & $\begin{array}{c}0.819 \\
(0.166) \\
\end{array}$ \\
\hline $\mathrm{Q}^{2}$ & & & & $\begin{array}{c}0.458 \\
(0.731)\end{array}$ \\
\hline TAILLE & $\begin{array}{c}-0.234 * * * \\
(-8.11)\end{array}$ & $\begin{array}{c}-0.239 * * * \\
(-8.574)\end{array}$ & $\begin{array}{c}-0.464 \\
(-0.627) \\
\end{array}$ & $\begin{array}{c}-0.714 \\
(-0.888) \\
\end{array}$ \\
\hline DETTE & $\begin{array}{c}0.0007 \\
(0.195)\end{array}$ & $\begin{array}{l}0.0004 \\
(0.117)\end{array}$ & & \\
\hline $\mathrm{RD}$ & $\begin{array}{l}0.038 * * \\
(2.364) \\
\end{array}$ & $\begin{array}{l}0.027^{*} \\
(1.718) \\
\end{array}$ & & \\
\hline LIQUIDITE & & & $\begin{array}{l}0.334^{*} \\
(1.66)\end{array}$ & $\begin{array}{l}0.308^{*} \\
(1.55)\end{array}$ \\
\hline BETA & & & 1.48 & 0.944 \\
\hline
\end{tabular}

\footnotetext{
${ }^{1}$ Résultats disponibles par les auteurs et peuvent être communiqués sur demande
} 


\begin{tabular}{|c|c|c|c|c|}
\hline & & & $(0.532)$ & $(0.333)$ \\
\hline RSPECIFIQUE & & & 0.06 & 0.052 \\
& & & $(1.088)$ & $(0.949)$ \\
\hline DIVID & & & 0.187 & 0.191 \\
& & & $(0.377)$ & $(0.391)$ \\
\hline $\mathrm{R}^{2}$ & 0.273 & 0.325 & 0.04 & 0.07 \\
\hline F- statistic & 16.917 & 17.963 & 4.602 & 4.017 \\
(P-value) & $(0.00)$ & $(0.00)$ & $(0.00)$ & $(0.00)$ \\
\hline
\end{tabular}

* Significatif au seuil de $10 \%$ ** significatif au seuil de $5 \%$ *** significatif au seuil de $1 \%$

\section{Conclusion}

L’objectif de cet article était d'examiner les relations possibles entre différents types d'institutionnels et la performance financière. Nous avons distingué ses acteurs selon leurs comportements et les facteurs qui les influencent.

Les résultats corroborent que l'influence des institutionnels sur la performance dépend effectivement de leur comportement (actif ou passif). Plus précisément, ils ont confirmé que : -l'observation d'un comportement actif des institutionnels est plus probable avec l'augmentation du nombre de facteurs d'influence d'un tel comportement, et influence par conséquence favorablement la performance des firmes,

- une relation bidirectionnelle existe entre les différents types d'investisseurs institutionnels et la performance des firmes.

Dans des recherches futures, nous proposons d'étudier l'impact de cette nouvelle typologie des investisseurs institutionnels sur les politiques et décisions stratégiques des firmes ainsi que d'examiner les interactions possibles de ces institutionnels avec d'autres types d'actionnaires.

\section{Références}

Agrawal A., Knoeber C.R., 1996, « Firm Performance and Mechanisms to Control Agency Problems between Managers and Shareholders », Journal of Financial and Quantitative Analysis, Vol. 31, $n^{\circ}$ 3, pp. 377-397.

Alexandre H., Paquerot M., 2000, " Efficacité des Structures de Contrôle et Enracinement des Dirigeants ", Finance Contrôle et Stratégie, Vol. 3, n 2, pp. 5-29.

Atanasov V., 2005, « How Much Value can Blockholders Tunnel? Evidence from the Bulgarian Mass Privatization Auctions », Journal of Financial Economics, 76, pp. 191-234. 
Black B.S., 1990, « Shareholder Passivity Re-examined », Michigan Law Review, Vol.89, n³, pp. 520-608.

Brickley J.A., Lease R.C., Smith C.W., 1988, «Ownership Structure and Voting on Antitakeover Amendments », Journal of Financial Economics, 20, pp. 267-291.

Bushee B.J., 1998, " The Influence of Institutional Investors on Myopic R\&D Investment Behaviour », The Accounting Review, Vol. 73, n 3, pp. 305-333.

Chen X., Harford J., Li K.., 2007, «Monitoring: Which Institutions Matter? », Journal of Financial Economics, 86, pp. 279-305.

Cornett M.M., Marcus A.J., Saunders A., Tehranian H., 2007, « The Impact of Institutional Ownership on Corporate Operating Performance », Journal of Banking and Finance, 31, pp. 1771-1794.

Cornett M.M., Marcus A.J., Tehranian H., 2008, « Corporate Governance and Pay- for-Performance: the Impact of Earnings Management », Journal of Financial Economics, 87, pp. 357-373.

Demsetz H., 1983, «The Structure of Ownership and the Theory of the Firm », Journal of Law and Economics, Vol. 24, pp. 375-390.

Demsetz H., Villalonga B., 2001, « Ownership Structure and Corporate Performance », Journal of Corporate Finance, Vol. 7, n 3, pp. 209-233.

Dong M., Ozkan A., 2008, « Institutional Investors and Director Pay: An Empirical Study of UK Companies », Journal of Multinational Financial Management, Vol. 18, n¹, pp. 16-29.

Hausman J.A., 1978, « Specification Tests in Econometrics », Econometrica, 46, 6, pp. 1251-1271.

Jensen M.C., Meckling W.H., 1976, « Theory of the Firm: Managerial Behaviour, Agency Costs and Ownership Structure », Journal of Financial Economics, Vol. 3, n 4, pp. 305-360.

Khan, R., Dharwadkar, R., Brandes, P., 2005, «Institutional Ownership and CEO

Compensation: a Longitudinal Examination», Journal of Business Research, 58, pp.1078-1088.

Kochhar R., David P., 1996, «Institutional Investors and Firm Innovation: A Test of Competing Hypotheses », Strategic Management Journal, 17, pp. 73-84.

Maug E., 1998, "Large Shareholders as Monitors: is there a Trade-Off between Liquidity and Control? ", The Journal of Finance, 53, pp. 595-612.

McConnell J.J., Servaes H., 1990, "Additional Evidence on Equity Ownership and Corporate Value », Journal of Financial Economics, Vol. 27, n² 2, pp. 595-612.

Mînguez-Vera A., Martin-Ugedo A.F., 2007, « Does Ownership Structure Affect Value? A Panel Data Analysis for the Spanish Market », International Review of Financial Analysis, 16, pp. 81-98.

Morck R., Shleifer A., Vishny R.W., 1988, " Management Ownership and Market Valuation: An Empirical Analysis », Journal of Financial Economics, 20, pp. 293-315.

Pound J., 1988, « Proxy Contests and the Efficiency of Shareholder Oversight », Journal of Financial Economics, 20, pp. 237-265.

Shleifer A, Vishny R.W., 1986, " Large Shareholders and Corporate Control », Journal of Political Economy, Vol. 94, n 3, pp. 461-489.

Switzer L.N., Kelly C., 2006, « Corporate Governance Mechanisms and the Performance of SmallCap Firms in Canada », International Journal Business Governance and Ethics, Vol. 2, $\mathrm{n}^{\circ}$ 3, 4 , pp. 294-328.

Tsai H., Gu Z., 2007, « The Relationship between Institutional Ownership and Casino Firm Performance », Hospitality Management, 26, pp. 517-530. 